\title{
La relevancia de la música en el desarrollo lingüístico de los bebés
}

\section{Clément François}

Laboratoire Parole et Langage, UMR7309, CNRS, Aix-Marseille University, Institute for Language Communication and the Brain

clement.francois@univ-amu.fr

Recepció: 18/06/2019, acceptació: 23/09/2019

Resumen: En los últimos veinte años, una gran cantidad de estudios se ha centrado en entender los mecanismos cerebrales que soportan el aprendizaje tanto del lenguaje como de la música. Tanto el lenguaje como la música se desarrollan en el tiempo, basándose en elementos individuales que son los sonidos y que se combinan formando estructuras más complejas y largas siguiendo reglas específicas que permiten la construcción de expectativas. Las preguntas relativas a si la música puede mejorar la adquisición de la lengua materna o bien facilitar el procesamiento de aspectos específicos del lenguaje tienen un gran interés, tanto para la investigación fundamental, que nos informa sobre el funcionamiento general del cerebro, como para la investigación más clínica, usando la música como herramienta de diagnóstico o de rehabilitación para niños con trastornos de aprendizaje. En este artículo reviso las relaciones entre la percepción del lenguaje y de la música en los primeros años de vida, describiendo las trayectorias de desarrollo de ambas habilidades perceptivas. También presento las pocas evidencias que demuestran un efecto positivo de una estimulación musical temprana sobre el procesamiento del lenguaje, poniendo énfasis en el papel del canto en los primeros pasos de la adquisición de la lengua materna.

Palabras claves: música, lenguaje, cerebro, infancia

\section{La rellevància de la música en el desenvolupament lingüístic dels nadons}

Resum: En els darrers vint anys, una gran quantitat d'estudis s'ha centrat en entendre els mecanismes cerebrals que suporten l'aprenentatge tant del llenguatge com de la música. Tant el llenguatge com la música es desenvolupen en el temps, basant-se en elements individuals que són els sons i que es combinen formant estructures més complexes i llargues seguint regles específiques que permeten la construcció d'expectatives. Les preguntes relatives a si la música pot millorar l'adquisició de la llengua materna o bé facilitar el processament d'aspectes específics del llenguatge tenen un gran interès, tant per a la investigació fonamental, que ens informa sobre el funcionament general del cervell, com per a la investigació més clínica, usant la música com a eina de diagnòstic o de rehabilitació per a nens amb trastorns d'aprenentatge. En aquest article reviso les relacions entre la percepció del llenguatge i de la música en els primers anys de vida, descrivint les trajectòries de desenvolupament d'ambdues habilitats perceptives. També presento les poques evidències que demostren un efecte positiu d'una estimulació musical primerenca sobre el processament del llenguatge, posant èmfasi en el paper del cant en els primers passos de l'adquisició de la llengua materna.

Paraules clau: música, llenguatge, cervell, infancia 


\section{The Relevance of Music in the Babie's Linguistic Development}

Abstract: In the last twenty years, a large number of studies have focused on understanding the brain mechanisms that support the learning of both language and music. Both language and music develop over time, based on individual elements that are sounds and which are combined to form more complex and longer structures following specific rules that allow the construction of expectations. Questions regarding whether music can improve the acquisition of the mother tongue or facilitate the processing of specific aspects of the language are of great interest, both for fundamental research, which informs us about the general functioning of the brain, and for more clinical research, using music as a diagnostic or rehabilitation tool for children with learning disorders. In this article I review the relations between the perception of language and music in the first years of life, describing the developmental trajectories of both perceptive abilities. I also present the few evidences that demonstrate a positive effect of an early musical stimulation on language processing, emphasizing the role of singing in the first steps of the acquisition of the mother tongue.

Keywords: music, language, brain, childhood

\section{INTRODUCCIÓN}

Durante los últimos 20 años, los avances en neurociencia, psicología del desarrollo y ciencias cognitivas han permitido entender mejor como se desarrolla el cerebro de los bebés y como adquieren su lengua materna (Dehaene-Lambertz y Spelke 2015). Estos progresos considerables permitirán una mejor detección y tratamiento de problemas graves de salud pública, como los trastornos del desarrollo. Recientemente, se ha desarrollado un conjunto de investigaciones con el objetivo de usar la música como una herramienta para estudiar la plasticidad cerebral relacionada con ciertas habilidades cruciales para un desarrollo lingüístico y cognitivo óptimo. Gracias a sus características estructurales la música ciertamente tiene un papel importante en el desarrollo. En este artículo reviso las relaciones entre la percepción del lenguaje y de la música en los primeros años de vida, describiendo las trayectorias de desarrollo de ambas habilidades perceptivas. También presento las pocas evidencias que demuestran un efecto positivo de una estimulación musical temprana sobre el procesamiento del lenguaje, poniendo énfasis en el papel del canto en los primeros pasos de la adquisición de la lengua materna.

\section{El DESARRollo PRECOZ DE LA PERCEPCión DEL LENGUAJE Y DE LA MÚSICA}

\subsection{Antes del nacimiento}

Uno se podría preguntar por qué es relevante estudiar las relaciones entre las capacidades musicales y el desarrollo lingüístico en bebés. En primer lugar, hay que considerar que entre los primeros sonidos que percibe el feto en el útero están las respiraciones de su madre y los latidos de su corazón. Desde el momento en que el feto puede oír (i.e. a partir de las 21 semanas de gestación), estos sonidos constituyen su primera experiencia con el ritmo. Durante este período prenatal, además de estos ritmos fisiológicos, el feto puede percibir también los sonidos de su entorno directo, principalmente las frecuencias graves, las que forman las melodías del lenguaje, es decir, la prosodia. Al nacer, ocurre un gran cambio perceptivo, el oído de los bebes cambia de una percepción auditiva en un medio líquido, basada únicamente en la transmisión de la 
información acústica por el líquido amniótico, a una percepción auditiva aérea, basada principalmente en la transmisión de la información acústica por el aire. Este cambio en el tipo de audición tiene consecuencias sobre el rango de frecuencias de los sonidos que el bebé puede percibir, tanto a nivel lingüístico como a nivel musical (Brandt et al. 2012).

\subsection{Durante los primeros años de vida}

Es interesante observar que, durante su primer año de vida, el bebé mostrará un incremento gradual en su sensibilidad auditiva, tanto frente al lenguaje como a la música. Durante los primeros meses de vida, se considera generalmente que el bebé es «ciudadano del mundo» porque tiene la capacidad de discriminar tanto contrastes fonéticos nativos (i.e. sonidos pertenecientes a la lengua de su entorno) como contrastes no-nativos. El incremento gradual de esa sensibilidad fonética se reflejará, por un lado, en mejoras en el desarrollo de su capacidad para discriminar los contrastes nativos, es decir, aquellos contrastes fonéticos presentes en el repertorio fonológico de su lengua materna y, por el otro, en una reducción de su sensibilidad frente a los contrastes no-nativos, es decir, los que pertenecen a lenguas no familiares y que no están presentes en el repertorio de la lengua materna (Kuhl 2004). Además de un aumento en la sensibilidad hacia los contrastes fonéticos nativos, se produce también un fenómeno muy parecido con los sonidos musicales. Por ejemplo, tras nacer, el bebé puede percibir no sólo cambios de tonos en melodías que pertenecen al sistema musical de su entorno, sino también cambios de tonos en melodías que pertenecen a un sistema musical diferente, no familiar, al que no ha estado expuesto previamente (Lynch et al. 1990). Un fenómeno similar ocurre con la percepción del ritmo: antes de los seis meses, el bebé será capaz de detectar cambios rítmicos complejos que no están presentes en su sistema musical (Hannon y Trehub 2005). De hecho, bajo la influencia que ejerce la exposición al entorno cultural lingüístico y musical, el bebé perderá gradualmente su sensibilidad a estos diferentes parámetros hasta que su oído «se afine» a su entorno. Finalmente, a los doce meses, el bebé se comportará perceptivamente como un adulto y perderá la capacidad de detectar las notas falsas dentro de melodías de un sistema de música extranjero o no-familiar (Lynch y Eilers 1992).

\section{LA RELEVANCIA DE LA MÚSICA EN EL PROCESAMIENTO DEL LENGUAJE}

\subsection{Las bases cerebrales del procesamiento del lenguaje y de la música}

Desde los trabajos pioneros de P. Broca y C. Wernicke en el siglo XIX, las posteriores investigaciones en neurociencias han permitido conocer mejor las estructuras cerebrales que comparten el procesamiento del lenguaje y de la música. En primer lugar, tanto el lenguaje como la música requieren la transmisión de la información neuronal a través de las vías auditivas, desde la cóclea al córtex auditivo, pasando por estructuras subcorticales como el colículo inferior. En este sentido existen varios estudios que han desvelado que las características acústicas de los sonidos del habla, así como las de los sonidos musicales, están codificadas a nivel subcortical con gran precisión por las neuronas del colículo inferior (Anderson et al. 2015, Kraus y Nicol 2005). Otros estudios, usando técnicas de resonancia magnética funcional, han podido demostrar que, independientemente de que el tipo de estímulo sea lingüístico o musical, tanto el córtex auditivo primario, como el secundario y el asociativo se activan, y esas activaciones se 
observan tanto en adultos como en bebés (Perani et al. 2010 Schön et al. 2010). Además, varios estudios han desvelado que el procesamiento de errores de naturaleza semántica o sintáctica, tanto en niños como en adultos, implica la activación específica de estructuras cerebrales como la corteza frontal inferior (Brown et al. 2006, Koelsch et al. 2005). Por lo tanto, existen en la literatura varias evidencias claras que demuestran que el procesamiento del lenguaje y de la música involucran redes cerebrales similares, con un alto nivel de solapamiento.

\subsection{La relevancia de la música en el desarrollo de las habilidades perceptivas del bebé}

Partiendo de investigaciones empíricas y teóricas destacadas en neurociencia y psicología experimental en los últimos 50 años, se ha formulado la hipótesis según la cual la práctica de un instrumento musical podría impactar de manera positiva en el procesamiento del lenguaje (Patel 2011, Besson et al. 2011). Hay dos estudios recientes, publicados en prestigiosas revistas, que han descrito el efecto positivo de intervenciones musicales tempranas durante el primer año de vida (Zhao y Kuhl 2016, Ortiz-Mantilla et al. 2019). De manera más específica, Zhao y Kuhl (2016) estudiaron una muestra de 47 bebés de nueve meses, monolingües, criados en Estados Unidos. Estos bebés fueron divididos en dos grupos, unos participaron en doce sesiones de quince minutos de estimulación musical y los otros, el grupo control, hicieron doce sesiones de quince minutos de juegos sin ningún componente musical. Dos semanas después de estas intervenciones, los investigadores, con el uso de la magnetoencefalografía, midieron la actividad cortical en respuesta a estímulos musicales y lingüísticos que contenían violaciones de su estructura rítmica. En comparación con el grupo control, los bebés que recibieron la estimulación musical presentaron una mayor actividad cortical, tanto en respuesta a secuencias musicales como a secuencias lingüísticas. Estos resultados son importantes por varias razones. Primero, a nivel metodológico, la inclusión de un grupo control permite especificar el papel de la intervención musical en relación con los cambios fisiológicos observados después de un mes de exposición a estímulos musicales. Segundo, los bebés que recibieron la intervención musical no sólo tenían mayor actividad en respuesta a violaciones de secuencias musicales, sino que tenían también una mayor actividad en respuesta a violaciones de secuencias lingüísticas desconocidas (no familiares). Este último resultado apunta a un efecto de transferencia o de generalización del procesamiento de la música al procesamiento del lenguaje. Finalmente, es interesante destacar también el valor del uso de medidas neurofisiológicas en este estudio realizado a una edad temprana, pues no precisan de ninguna respuesta conductual por parte del bebé y tampoco requieren que los bebés presten atención a los estímulos de la prueba.

Otro artículo reciente ha podido desvelar que una estimulación acústica interactiva temprana, consistente en una sesión semanal durante seis semanas consecutivas, entre los cuatro y los seis meses de edad, facilita el procesamiento acústico en bebés de nueve meses (Ortiz-Mantilla et al. 2019). En ese estudio fueron reclutados dos grupos de bebés de cuatro a seis meses de edad. Un grupo recibió estimulación acústica interactiva temprana que consistía en aprender la asociación entre una serie de estímulos auditivos y un video de recompensa, mientras que el otro recibió los mismos estímulos auditivos, pero sin estar asociados a un video de recompensa. A los nueve meses, estos bebés participaron en una sesión experimental con registro de medidas electroencefalográfícas, durante la cual los bebés fueron expuestos a una serie de sílabas artificiales (e.g. /da/), definidas como estímulos estándar dentro de la serie, y a otras silabas con un tiempo del inicio de la sonoridad (TIS, en español; Voice-Onset-Time, VOT, en inglés) diferente (e.g. 
/ta/). Los resultados fueron muy interesantes: se demostró que los bebés expuestos a la estimulación acústica interactiva presentaban una mayor respuesta oscilatoria (Highgamma $>70 \mathrm{~Hz}$ ) a las sílabas estándar que los bebes del grupo control, sugiriendo un mejor procesamiento de los sonidos del habla. Además, los bebés expuestos a la estimulación acústica interactiva presentaban también un incremento de la respuesta oscilatoria (left-lateralized gamma $<70 \mathrm{~Hz}$ ) a las sílabas con un cambio en el tiempo del inicio de la sonoridad, sugiriendo una mejor habilidad de discriminación entre sílabas en el grupo de estimulación acústica interactiva. Sin embargo, en estos dos estudios no se obtuvieron medidas neurofisiológicas antes de la intervención, con lo cual no se puede descartar la posibilidad de que existieran diferencias entre los dos grupos, antes de la intervención, que explicasen los resultados obtenidos. Así pues, estos resultados, aunque con limitaciones, apuntan a un impacto positivo de una intervención musical temprana sobre las habilidades perceptivas del lenguaje.

\subsection{La relevancia del canto para la segmentación del habla en recién nacidos}

Si una buena percepción de los sonidos del habla es algo crucial para el buen desarrollo lingüístico de los bebés, otra habilidad primordial para la correcta adquisición de su lengua materna es la capacidad de segmentación del habla, fenómeno que tiene lugar aproximadamente a partir del segundo semestre de vida. Antes de producir las primeras palabras o incluso antes de balbucear, el lactante puede reconocer fácilmente algunas palabras familiares aisladas, e incluso logra segmentarlas del flujo continuo del habla. Esta capacidad para segmentar y extraer palabras de la señal continua del habla se considera fundamental en la adquisición del lenguaje, y un requisito necesario para el correcto desarrollo de las habilidades lingüísticas (Newman et al. 2006).

Los índices prosódicos, como el acento léxico, las pausas, la entonación, o la melodía del lenguaje, son cruciales para la segmentación de las palabras, tanto en adultos (Cunillera et al. 2006), como en bebés (Johnson et al. 2001). En colaboración con los investigadores L. Bosch y A. Rodríguez-Fornells de la Universidad de Barcelona, diseñamos recientemente un estudio electrofisiológico para investigar el posible impacto positivo de las claves o índices prosódicos en el procesamiento inicial del habla y en su proceso de segmentación, basándonos en la hipótesis de la iniciación prosódica o prosodic bootstrapping en inglés (Christophe y Dupoux 1996). La iniciación prosódica se refiere a la hipótesis según la cual los bebés usan las características prosódicas de la lengua materna (modulaciones de tono, de ritmo, de intensidad y otros parámetros acústicos del habla) como claves o pistas (cues, en inglés) para identificar y aprender las propiedades estructurales del lenguaje, como por ejemplo la sintaxis. Así pues, usando un paradigma novedoso donde medimos la actividad electro-encefalográfica de bebés de dos días mientras estaban expuestos a un lenguaje artificial hablado o cantado. Comparando la condición cantada con la condición hablada, pudimos estudiar las modulaciones de actividad cerebral relacionadas con el aprendizaje de regularidades (fase de aprendizaje), así como el propio resultado del proceso de aprendizaje (fase de prueba implícita). En concreto, en la condición hablada, los bebés fueron expuestos durante apenas cuatro minutos a un flujo continuo de sílabas artificiales formando cuatro pseudopalabras trisilábicas, con la predictibilidad que se da entre sílabas consecutivas (probabilidades transicionales entre sílabas de una misma palabra) como único índice útil a utilizar para poder segmentar el flujo continuo de habla. A continuación, los bebés fueron expuestos al mismo flujo continuo, pero esta vez con palabras «ilegales» (i.e. palabras que no respetaban la secuencia silábica con la que se habían familiarizado los bebés en la fase de 
aprendizaje; la «ilegalidad» se debía al intercambio de posición entre la primera y la tercera sílabas) incluidas en el flujo. En la condición cantada, los bebés fueron expuestos a otro flujo continuo de palabras trisilábicas en las cuales cada sílaba estaba siempre asociada a un tono musical específico, creando así un solapamiento entre la estructura silábica y la estructura melódica. De la misma manera que en la condición hablada, después de esta fase de aprendizaje los bebes fueron expuestos al flujo continuo de sílabas cantadas, pero esta vez con palabras «ilegales» (i.e. que no respetaban el orden secuencial de la estructura silábica debido a una inversión entre la primera y la tercera sílaba) incluidas dentro del flujo.

El análisis de la actividad cerebral recogida durante la fase de aprendizaje (i.e. los potenciales evocados por las pseudopalabras trisilábicas durante la primera y la segunda mitad de la fase de aprendizaje) reveló la existencia de diferentes patrones de actividad cerebral para las secuencias habladas y cantadas. En el caso de la condición hablada, observamos que las pseudopalabras inducían una positividad durante la primera mitad de la fase de aprendizaje y una negatividad durante la segunda mitad. Al contrario, en la condición cantada observamos que las pseudopalabras inducían una negatividad durante la primera mitad de la fase de aprendizaje y una positividad durante la segunda mitad. Estos resultados recordaban claramente los de estudios previos en adultos demostrando un vínculo entre la dinámica cerebral durante la fase de aprendizaje de palabras y el reconocimiento de estas palabras durante una prueba conductual (Cunillera et al. 2009, De Diego Balaguer et al. 2007, François et al. 2014). Una posible explicación de esta diferencia de dinámicas es que, en la condición cantada, las palabras contenían cambios de contorno prosódico e intervalos que coincidían con las fronteras de las palabras, lo que podría haber facilitado la agrupación de sílabas en virtud de las propiedades gestálticas del canto (Schön et al. 2008).

El análisis de la actividad cerebral durante las fases de prueba implícita nos permitió observar modulaciones de la actividad cerebral reflejando la detección de violaciones de la estructura silábica en la condición cantada, pero ninguna en la condición hablada. Mientras que en la condición cantada se observaba una respuesta cerebral específica para las secuencias ilegales, no era el caso en la condición hablada en la cual los potenciales evocados no diferenciaban las secuencias legales de las ilegales. Este resultado sugiere que la condición cantada había permitido la formación de huellas mnésicas suficientemente robustas para generar expectativas que permiten la detección de violaciones de la estructura silábica. Estos resultados a dos días de vida nos permitieron demostrar el efecto positivo del canto sobre las capacidades de segmentación del habla continua en recién nacidos (François et al. 2017). Finalmente, el estudio contemplaba el seguimiento de este grupo de neonatos hasta la edad de dieciocho meses con la obtención de datos neuropsicológicos mediante las escalas Bayley-III y medidas de desarrollo lingüístico en diferentes etapas del desarrollo temprano mediante la versión española del Inventario de Desarrollo Comunicativo MacArthur (López-Ornat et al. 2005). Los resultados de esta parte longitudinal, realizada con una sub-muestra del grupo inicial, revelaron que la dinámica cerebral recogida durante la fase de aprendizaje para las secuencias cantadas (i.e. la amplitud media entre 200 y $500 \mathrm{~ms}$ durante la segunda mitad de la fase aprendizaje menos la amplitud media entre 200 y $500 \mathrm{~ms}$ durante la primera mitad de la fase de aprendizaje) predecía el vocabulario expresivo a los 18 meses, medido mediante la versión española del Inventario MacArthur. Este último resultado sugiere que las diferencias individuales tempranas, a nivel electrofisiológico, en el procesamiento de la prosodia podrían ser un buen indicador del desarrollo lingüístico posterior. 


\section{CONCLUSiONES}

En conclusión, las dinámicas de desarrollo de las habilidades lingüísticas y musicales parecen estar más íntimamente entrelazadas de lo que cabría esperar. En un futuro próximo, los resultados en el campo de las neurociencias y la psicología experimental del desarrollo podrán ayudar a entender el papel de la música en el desarrollo lingüístico temprano en niños sanos. Como además se ha sugerido en un artículo de revisión (François et al. 2015), los resultados de estudios que demuestran la eficacia de las intervenciones musicales son importantes para un mejor tratamiento de los trastornos del aprendizaje del lenguaje, como la dislexia (Flaugnacco et al. 2015, Frey et al. 2019).

\section{AgRadeCIMIENTOS}

Muchas gracias a Toni Cunillera y Laura Bosch por corregir el manuscrito.

\section{REFERENCIAS BIBLIOGRÁFICAS}

Anderson, Samira; Parbery-Clark, AleXandra; White-Schwoch, Travis; Kraus, Nina (2015). Development of subcortical speech representation in human infants. J. Acoust. Soc. Am, 137(6), 3346-3355.

Besson, Mireille; Chobert, Julie; Marie, Céline (2011). Transfer of Training between Music and Speech: Common Processing, Attention, and Memory. Frontiers in Psychology. 2:94.

Brandt, ANThony K.; Slevc, Robert; Gebrian, Molly (2012). Music and early language acquisition. Frontiers in Psychology, 3, 327.

Brown, Steven; Martinez, Michael J.; Parsons, Lawrence M. (2006). Music and language side by side in the brain: a PET study of the generation of melodies and sentences. Eur. J. Neurosci. 23, 2791-2803.

Christophe, Anne; Dupoux, Emmanuel (1996). Bootstrapping lexical acquisition: the role of prosodic structure. The Linguistic Review, 13(3-4), 383-412.

Cunillera, Toni; Toro, Juan M.; Sebastián-Galles, Núria; RodríGuez-Fornells, ANTONI (2006). The effects of stress and statistical cues on continuous speech segmentation: an event-related brain potential study. Brain Research, 1123(1), 168-178.

Cunillera, Toni et al. (2009). Time course and functional neuroanatomy of speech segmentation in adults. NeuroImage 48(3), 541-553.

De Diego Balaguer, Ruth; Toro, Juan M.; Rodríguez-Fornells, Antoni; BachoudLÉvi, ANNe CATHERINe (2007). Different Neurophysiological Mechanisms Underlying Word and Rule Extraction from Speech. PLoSONE, 2 (11), e1175.

Dehaene-Lambertz, Ghislaine; Spelke, Elizabeth (2015). The infancy of the human brain. Neuron, 88(1), 93-109.

Flaugnacco, Elena; Lopez, Luisa; Terribili, Chiara; Montico, Marcella; Zoia, STEFANIA; SCHÖN, DANIELE (2015). Music training increases phonological awareness and reading skills in developmental dyslexia: a randomized control trial. PloSONE, 10(9), e0138715.

Francois, Clément; Teixido, Maria; Takerkart, Sylvain; Agut, Thaïs; Bosch, Laura; RODRÍGUEZ-FoRNELLS, ANTONI (2017). Enhanced neonatal brain responses to sung streams predict vocabulary outcomes by age 18 months. Scientific Reports, 7(1), 12451 
Francois, Clement; Grau-SAnchez, Jennifer; Duarte, Esther; Rodriguez-FornelLs, ANTONI (2015). Musical training as an alternative and effective method for neuroeducation and neuro-rehabilitation. Frontiers in psychology, 6, 475.

Francois, Clément; Jaillet, Florent; Takerkart, Sylvain; Schön, Daniele (2014). Faster sound stream segmentation in musicians than in nonmusicians. PloSONE, 9(7):e101340.

Frey, Aline; François, Clement; Chobert, Julie; Velay, Jean-Luc; Habib, Michel; Besson, Mireille (2019). Music Training Positively Influences the Preattentive Perception of Voice Onset Time in Children with Dyslexia: A Longitudinal Study. Brain sciences, 9(4), 91.

Hannon, Erin E.; Trehub, Sandra E. (2005). Tuning in to musical rhythms: infants learn more readily than adults. Proc. Natl. Acad. Sci. U.S.A, 102, 12639-12643.

Johnson, Elizabeth K.; JusczyK, Peter W. (2001). Word segmentation by 8-montholds: When speech cues count more than statistics. J. Mem. and Lang, 44, 548-567.

Koelsch, Stefan; Fritz, Thomas; Schulze, Katrin; Alsop, D.; Schlaug, Gottrried (2005). Adults and children processing music: an fMRI study. Neuroimage, 25(4), 10681076.

Kraus, Nina; Nicol, Trent (2005). Brainstem origins for cortical 'what' and 'where' pathways in the auditory system. Trends Neurosci, 28(4), 176-181.

KuHL, PATRICIA K. (2004). Early language acquisition: cracking the speech code. Nature Review Neuroscience, 5, 831-843.

López-Ornat, Susana; Gallego, Carlos; Gallo, Pilar; Karousou, Alexandra; MARISCAL, SONIA; MARTíneZ, MARÍa (2005). Inventario de Desarrollo Comunicativo MacArthur (adaptación española). Madrid: TEA Ediciones.

Lynch, Michael P.; Eilers, Rebecca E.; Kimbrough Oller, D.; Urbano, Richard C. (1990). Innateness, experience, and music perception. Psychological Science, 1, 272-276.

Lynch, Michael P.; Eilers, Rebecca E. (1992). A study of perceptual development for musical tuning. Perception and Psychophysic, 52, 599-608.

Newman, Rochelle; Ratner, Nan Bernstein; Jusczyk, Ann Marie; Jusczyk, Peter W.; DOW, KaTHY Ayala (2006). Infants' early ability to segment the conversational speech signal predicts later language development: a retrospective analysis. Developmental Psychology, 42(4), 643-655.

Ortiz-Mantilla, Silvia; Realpe-Bonilla, Teresa; Benasich, April A. (2019). Early Interactive Acoustic Experience with Non-speech Generalizes to Speech and Confers a Syllabic Processing Advantage at 9 Months. Cerebral Cortex, 29(4), 1789-1801.

Patel, ANiRuddh D. (2011). Why would Musical Training Benefit the Neural Encoding of Speech? The OPERA Hypothesis. Frontiers in Psychology, 2, 142.

Perani, Daniela; Saccuman, Maria Cristina; Scifo, Paola; Spada, Danilo; Andreolli, Guido; Rovelli, Rosanna; Baldoli, Cristina; Koelsch, Stefan (2010). Functional specializations for music processing in the human newborn brain. Proc. Natl. Acad. Sci. U.S.A, 107, 4758-4763.

Schön, Daniele; Boyer, Maud; Moreno, Sylvain; Besson, Mireille; Peretz, Isabelle; KOLINSKY, RÉGINE (2008). Songs as an aid for language acquisition. Cognition, 106(2), 975-983.

Schön, Daniele; Gordon, Reyna; Campagne, Aurélie; Magne, Cyrille; Astesano, Corine; ANTON, JEAN-LuC; BESSON, MireILle (2010) Similar cerebral networks in language, music and song perception. Neuroimage, 51, 450-461. 
ZhaO, T. Christina; Kuhl, Patricia K. (2016). Musical intervention enhances infants' neural processing of temporal structure in music and speech. Proc. Natl. Acad. Sci. U.S.A, 113(19), 5212-5217. 